\title{
David Lussier and Pierre Beaulieu (eds): Adjuvant analgesics
}

\author{
Oxford American Pain Library - Oxford University Press, 2015, \\ ISBN 978-0-19-989181-8
}

\author{
Sarika Alisic, MD
}

Received: 15 November 2015/Revised: 17 November 2015/Accepted: 1 December 2015/Published online: 11 December 2015

(c) Canadian Anesthesiologists' Society 2015

The practice of pain medicine has evolved to include analgesics from a myriad of pharmacologic classes. As we elucidate the complex pathways involved in pain transduction, transmission, modulation, and perception, the goal is to prescribe analgesics with specific targets. In Adjuvant Analgesics, the editors David Lussier and Pierre Beaulieu aim to summarize the literature and provide guidance on the analgesics with the strongest evidence for current use.

The evidence-informed approach to this textbook is a major strength that allows for brevity in its presentation. Indeed, at only 145 pages, the pocketbook is a good resource for physicians who are already versed in the practice of pain medicine, although it may not serve well as an introductory level book for medical students or other trainees.

The book is divided into ten concise chapters. It begins with a brief introduction into the theory of pain management and then focuses on the different classes of adjuvant analgesics. This is a multi-author textbook, and clearly, the authors have spent considerable effort in researching their focused areas of interest. The first two chapters provide a comfortable framework to introduce the concept of multidisciplinary care and adjuvant analgesics. These two chapters incorporate multiple classification tables of analgesics, and interestingly, this highlights the fact that there is no universally accepted classification system used by pain physicians. For completeness, it may have been beneficial to include interventional procedures as a fourth step in the World Health Organization analgesic

S. Alisic, MD (ه)

The Ottawa Hospital, University of Ottawa, Ottawa, ON, Canada

e-mail: salisic@toh.on.ca ladder. In addition, and although not an objective of the textbook, a section on pain pathophysiology would have grounded some of the terms interspersed throughout the textbook, including the concepts of central and peripheral sensitization.

This book provides an organized synthesis of the applicable research in each category of adjuvant analgesics. The end result is a very useful guide for clinicians attempting to make clinical decisions in the face of a multitude of research studies with small sample sizes and/or without a comparator. The summary tables of available evidence are particularly helpful, especially the table in chapter 4 on anticonvulsants, which also contains information on dose titrations and side effects. The authors include numbers needed to treat when comparing adjuvant analgesics within a specific category and pain indication (e.g., antidepressants in chapter 3 and anticonvulsants in chapter 4), further highlighting the textbook's clinical utility. Chapter 10, which is focused on drug interactions between diverse adjuvant analgesics, is particularly well written and contains several comprehensive and clinically useful tables.

A drawback of many multi-author textbooks, and this one is no exception, is some repetition between the various sections. As examples, the discussion of cannabinoids in chapter 5 is also addressed in chapter 9 and there are also duplicate tables (tables 2.3 and 9.2.1). The pertinent information is presented within the context of the respective sections; however, the textbook could be improved with further editing to avoid this repetition. It was also challenging to understand how the subsections of chapter 9 are interrelated (9.1 Neuropathic Pain; 9.2 Cancer-related Pain; 9.3 Rheumatic Pain and Fibromyalgia; 9.4 Acute Postoperative Pain), and an 
explanation for their inclusion under a singular chapter would have been useful for the reader.

Overall, this textbook provides a thorough review and analysis of the pain literature and highlights studies that are of limited scope and where further research is required. In outlining and succinctly summarizing the vast amount of available literature, it remains clear that a plethora of information is still unknown and that very often the decision surrounding drug selection is still largely empirical and based on physician anecdotal experience. Books such as this aim to reduce this practice, but there is considerable room for continued improvement in our decision making.

Compliance with ethical standards

Conflicts of interest None declared. 